Mathematical Modelling AND ANALysis

Volume 18 Number 1, February 2013, 103-121

http://dx.doi.org/10.3846/13926292.2013.760011

(c) Vilnius Gediminas Technical University, 2013
Publisher: Taylor\&Francis and VGTU

http://www.tandfonline.com/TMMA

Print ISSN: 1392-6292

Online ISSN: 1648-3510

\title{
A Quadratic B-Spline Galerkin Approach for Solving a Coupled KdV Equation
}

\section{Selcuk Kutluay and Yusuf Ucar}

\author{
Inönü University, Faculty of Arts and Sciences \\ 44280 Malatya, Turkey \\ E-mail: selcuk.kutluay@inonu.edu.tr \\ E-mail(corresp.): yusuf .ucar@inonu.edu.tr
}

Received April 15, 2012; revised November 12, 2012; published online February 1, 2013

\begin{abstract}
In this paper, a quadratic B-spline Galerkin finite element approach is applied to one-dimensional coupled $\mathrm{KdV}$ equation in order to obtain its numerical solutions. The performance of the method is examined on three test problems. Computed results are compared with the exact results and also other numerical results given in the literature. A Fourier stability analysis of the approach is also done.
\end{abstract}

Keywords: coupled KdV equation, finite element method, Galerkin method, quadratic B-spline, Fourier stability analysis.

AMS Subject Classification: 65D07; 65N30; 74S05; 65L60.

\section{Introduction}

In this paper, we will consider the coupled $\mathrm{KdV}$ equation of the form

$$
\begin{aligned}
& U_{t}-6 a U U_{x}-2 b V V_{x}-a U_{x x x}=0, \quad c<x<d, t>0, \\
& V_{t}+3 U V_{x}+V_{x x x}=0, \quad c<x<d, t>0
\end{aligned}
$$

with the boundary conditions

$$
\begin{aligned}
& U(c, t)=0, \quad U(d, t)=0, \quad V(c, t)=0, \quad V(d, t)=0, \quad t>0, \\
& U_{x}(c, t)=0, \quad U_{x}(d, t)=0, \quad V_{x}(c, t)=0, \quad V_{x}(d, t)=0, \quad t>0
\end{aligned}
$$

and the initial conditions

$$
U(x, 0)=f(x), \quad V(x, 0)=g(x), \quad c<x<d,
$$

where $f(x)$ and $g(x)$ are the prescribed functions, $a$ and $b$ are arbitrary constants [13].

The coupled $\mathrm{KdV}$ equation used in this paper describes the interaction of two long waves with different dispersion relations. Thus, this coupled equation 
is related to most types of long waves having weak dispersion. Some internal acoustic and planetary waves arising in geophysical fluid mechanics can be given as examples [6].

The coupled KdV type equations have been the most important class of nonlinear evolution equations arising in physical sciences and engineering. Therefore, the coupled $\mathrm{KdV}$ type equations have been analytically considered by several authors: Hirota and Satsuma [13], and Tam et al. [27] have presented a coupled Korteweg-de Vries equation and obtained soliton solutions of the equation. Tian and Gao [28] have obtained the exact solutions to the Bogoyavlenskii coupled KdV equations via an auto-Backlund transformation with the application of the Painlevė analysis and computer algebra. Fan and Zhang [10] have obtained several kinds of exact solutions for a system of coupled KdV equations using an improved homogeneous balance method. Roy [20] has examined the bi-Hamiltonian structure of two coupled KdV equations proposed by Hirota and Satsuma [13], and Ito [17]. Zhu [30] has given a difference scheme for the periodic initial-boundary problem of the coupled KdV Equation. Cao et al. [7] have obtained more exact solutions for a new coupled MKdV equations by using a direct and efficient trigonometric function transform method based on the idea of the homogeneous balance method and for KdV equations by Miura transformation. Zhou et al. [29] have obtained the periodic wave solutions to a coupled KdV equations with variable coefficients by using F-expansion method which can be thought of as an over-all generalization of Jacobi elliptic function expansion method. Karasu and Kilic [18] have studied the Painleve property of coupled, non-autonomous KdV type of systems and obtained the conditions under which the systems pass the Painleve test for integrability. Qian and Tian [24] have found the single soliton solutions for a coupled KdV equation using the non-local Lie-Backlund transformation theorem to the trivial zero solution. Inan [14] has given some exact solutions of the coupled KdV equation by using the generalized tanh function method. Ma and Zhu [21] have obtained some new exact solutions of the coupled KdV equations via the Jacobian elliptic function expansion approach and Hermite transformation. Assas [5] has applied He's variational iteration method to solve the non-linear coupled-KdV equation based on the use of Lagrange multipliers for identification of optimal value of a parameter in a functional. Abbasbandy [1] has used an analytical technique, namely the homotopy analysis method, to solve a generalized Hirota-Satsuma coupled KdV equation. Al-Khaled et al. [3] have applied both the tanh and the He's variational iteration methods for analytical study for the nonlinear coupled KdV equations. Mokhtari and Mohammadi [22] have decomposed a coupled system of nonlinear partial differential equations into a set of algebraic equations as well as an ordinary differential equation and then solved by using Exp-function method.

The coupled KdV type equations have been also considered numerically by some authors: Fan [9] has obtained numerical solutions of the coupled KdV equation by using a Riccati equation involving a parameter and its solutions to replace the tanh-function in the tanh method. Halim et al. [11] have introduced a numerical method for general coupled KdV systems valid for solving Cauchy problems for arbitrary number of equations with arbitrary constant coefficients. 
Halim and Leble [12] have selected a second covariant equation to form Lax pair of a coupled KdV-MKdV system and introduced a numerical method for general KdV-MKdV system. Kaya and Inan [19] have found the explicit and numerical traveling wave solutions for a coupled KdV equation and a coupled MKdV equation by using the decomposition method with the help of symbolic computation. Alvarez-Samaniegoa and Carvajalb [4] have studied the locally well-posed conditions of coupled KdV equations for some systems. Ismail [16] has set up a numerical method for solving the coupled $\mathrm{KdV}$ equation based on the collocation method with quintic B-spline finite elements to simulate the solution of coupled KdV equation. Siraj-ul-Islam et al. [15] have formulated a simple classical radial basis functions collocation method for the numerical solution of the coupled KdV equations. Rady et al. [2] have considered the system of coupled $\mathrm{KdV}$ equations and established the transformation which turns the coupled $\mathrm{KdV}$ equations into the single nonlinear partial differential equation, then they obtained an auto-Backlund transformation and lax pairs using the extended homogeneous balance method. Biswas and Ismail [6] have used solitary wave ansatz to carry out the integration of the coupled KdV equation with power law nonlinearity, and then supplemented their results by numerical simulations. Recently, Rashid and Ismail [25] have obtained error estimates of spectral collocation method for the coupled $\mathrm{KdV}$ equations and presented their numerical solutions.

In this paper, we have applied a Galerkin quadratic B-spline finite element method to the one dimensional coupled $\mathrm{KdV}$ equation given by (1.1) with a set of initial and boundary conditions to obtain its numerical solutions.

\section{The Finite Element Solution}

The finite interval $[c, d]$ is divided into $N$ finite elements of equal length $h$ by the knots $x_{i},(i=0, \ldots, N)$ such that $c=x_{o}<x_{1}<\cdots<x_{N-1}<x_{N}=d$ and $h=\Delta x=x_{i}-x_{i-1}=(d-c) / N$. The quadratic B-splines $Q_{m}(x)$, $m=-1, \ldots, N$, which form a basis for functions over the interval $[c, d]$ are defined as $[23]$

$$
Q_{m}(x)=\frac{1}{h^{2}} \begin{cases}\left(x-x_{m-1}\right)^{2}, & {\left[x_{m-1}, x_{m}\right],} \\ \left(x-x_{m-1}\right)^{2}-3\left(x-x_{m}\right)^{2}, & {\left[x_{m}, x_{m+1}\right],} \\ \left(x-x_{m-1}\right)^{2}-3\left(x-x_{m}\right)^{2}+3\left(x-x_{m+1}\right)^{2}, & {\left[x_{m+1}, x_{m+2}\right],} \\ 0, & \text { otherwise. }\end{cases}
$$

So global approximations $U_{N}(x, t)$ and $V_{N}(x, t)$ to the exact solutions $U(x, t)$ and $V(x, t)$ are respectively sought in terms of the quadratic B-splines as

$$
U_{N}(x, t)=\sum_{j=-1}^{N} \delta_{j}(t) Q_{j}(x), \quad V_{N}(x, t)=\sum_{j=-1}^{N} \sigma_{j}(t) Q_{j}(x),
$$

where $\delta_{j}$ and $\sigma_{j}$ are time dependent parameters to be determined from the boundary and weighted residual conditions. 
In terms of the local coordinate transformation $\xi=x-x_{m}, 0 \leq \xi \leq h$, the splines can be expressed as

$$
\begin{aligned}
& Q_{m-1} \\
& Q_{m} \\
& Q_{m+1}
\end{aligned}=\frac{1}{h^{2}}\left\{\begin{array}{l}
(h-\xi)^{2} \\
h^{2}+2 h \xi-2 \xi^{2}, \quad 0 \leq \xi \leq h \\
\xi^{2}
\end{array}\right.
$$

Since all other quadratic B-splines are zero over the element $\left[x_{m}, x_{m+1}\right]$, the approximation (2.1) over this element can be written in terms of the basis functions $(2.2)$ as

$$
U_{N}=\sum_{j=m-1}^{m+1} \delta_{j} Q_{j}, \quad V_{N}=\sum_{j=m-1}^{m+1} \sigma_{j} Q_{j} .
$$

Using Eqs. (2.2) and (2.3), the nodal values $U_{m}, V_{m}, U_{m}^{\prime}$ and $V_{m}^{\prime}$ at the knot $x_{m}$ are obtained in terms of time-dependent element parameters $\delta_{m}$ and $\sigma_{m}$ as

$$
\begin{aligned}
& U\left(x_{m}\right)=U_{m}=\delta_{m-1}+\delta_{m}, \quad V\left(x_{m}\right)=V_{m}=\sigma_{m-1}+\sigma_{m}, \\
& U^{\prime}\left(x_{m}\right)=U_{m}^{\prime}=\frac{2}{h}\left(\delta_{m}-\delta_{m-1}\right), \quad V^{\prime}\left(x_{m}\right)=V_{m}^{\prime}=\frac{2}{h}\left(\sigma_{m}-\sigma_{m-1}\right) .
\end{aligned}
$$

In the above equations and throughout this paper, the prime denotes differentiation with respect to $x$.

In the Galerkin method, basis functions given by (2.2) are used as weighting functions $W_{m}$. Thus, the weak form of Eq. (1.1) with boundary conditions (1.2) over each finite element $\left[x_{m}, x_{m+1}\right]$ leads to the integral equation

$$
\begin{aligned}
& \int_{x_{m}}^{x_{m+1}}\left(W U_{t}-6 a W U U_{x}-2 b W V V_{x}+a W_{x} U_{x x}\right) d x=0 \\
& \int_{x_{m}}^{x_{m+1}}\left(W V_{t}+3 W U V_{x}-W_{x} V_{x x}\right) d x=0
\end{aligned}
$$

By inserting Eqs. (2.2) and (2.3) into Eq. (2.4), we have

$$
\begin{aligned}
& \sum_{j=m-1}^{m+1}\left(\int_{0}^{h} Q_{i} Q_{j} d \xi\right) \dot{\delta}_{j}^{e}-6 a \sum_{k=m-1}^{m+1} \sum_{j=m-1}^{m+1}\left[\left(\int_{0}^{h} Q_{i} Q_{k}^{\prime} Q_{j} d \xi\right) \delta_{j}^{e}\right] \delta_{k}^{e} \\
& -2 b \sum_{k=m-1}^{m+1} \sum_{j=m-1}^{m+1}\left[\left(\int_{0}^{h} Q_{i} Q_{k} Q_{j}^{\prime} d \xi\right) \sigma_{j}^{e}\right] \sigma_{k}^{e}+a \sum_{j=m-1}^{m+1}\left(\int_{0}^{h} Q_{i}^{\prime} Q_{j}^{\prime \prime} d \xi\right) \delta_{j}^{e}=0 \\
& \sum_{j=m-1}^{m+1}\left(\int_{0}^{h} Q_{i} Q_{j} d \xi\right) \dot{\sigma}_{j}^{e}+3 \sum_{k=m-1}^{m+1} \sum_{j=m-1}^{m+1}\left[\left(\int_{0}^{h} Q_{i} Q_{k}^{\prime} Q_{j} d \xi\right) \delta_{j}^{e}\right] \sigma_{k}^{e} \\
& \quad-\sum_{j=m-1}^{m+1}\left(\int_{0}^{h} Q_{i}^{\prime} Q_{j}^{\prime \prime} d \xi\right) \sigma_{j}^{e}=0
\end{aligned}
$$


which can also be written in the matrix form as

$$
\begin{aligned}
& A^{e} \dot{\delta^{e}}-6 a B^{e}(\delta) \delta^{e}-2 b \widetilde{B}^{e}(\sigma) \sigma^{e}+a C^{e} \delta^{e}=0, \\
& A^{e} \dot{\sigma}^{e}+3 B^{e}(\delta) \sigma^{e}-C^{e} \sigma^{e}=0 .
\end{aligned}
$$

In the above equations and throughout the paper, the dot denotes differentiation with respect to $t, \delta^{e}=\left(\delta_{m-1}, \delta_{m}, \delta_{m+1}\right)$ and $\sigma^{e}=\left(\sigma_{m-1}, \sigma_{m}, \sigma_{m+1}\right)$ are the element parameters, and $A_{i j}^{e}, B_{i k j}^{e}, \widetilde{B}_{i k j}^{e}$ and $C_{i j}^{e}$ are the element matrices given by the following integrals:

$$
\begin{aligned}
& A_{i j}^{e}=\int_{0}^{h} Q_{i} Q_{j} d \xi, \quad B_{i k j}^{e}=\int_{0}^{h} Q_{i} Q_{k}^{\prime} Q_{j} d \xi \\
& \widetilde{B}_{i k j}^{e}=\int_{0}^{h} Q_{i} Q_{k} Q_{j}^{\prime} d \xi, \quad C_{i j}^{e}=\int_{0}^{h} Q_{i}^{\prime} Q_{j}^{\prime \prime} d \xi,
\end{aligned}
$$

where $i, j, k=m-1, m, m+1$. The element matrices (2.6) are calculated as

$$
\begin{aligned}
A_{i j}^{e}= & \int_{0}^{h} Q_{i} Q_{j} d \xi=\frac{h}{30}\left[\begin{array}{ccc}
6 & 13 & 1 \\
13 & 54 & 13 \\
1 & 13 & 6
\end{array}\right], \\
B_{i k j}^{e}= & \int_{0}^{h} Q_{i} Q_{k}^{\prime} Q_{j} d \xi=\frac{1}{30}\left[\begin{array}{ccc}
(-10,-19,-1) \delta^{e} & (8,12,0) \delta^{e} & (2,7,1) \delta^{e} \\
(-19,-54,-7) \delta^{e} & (12,0,-12) \delta^{e} & (7,54,19) \delta^{e} \\
(-1,-7,-2) \delta^{e} & (0,-12,-8) \delta^{e} & (1,19,10) \delta^{e}
\end{array}\right], \\
\widetilde{B}_{i k j}^{e}= & \int_{0}^{h} Q_{i} Q_{k} Q_{j}^{\prime} d \xi=\frac{1}{30}\left[\begin{array}{ccc}
(-10,8,2) \sigma^{e} & (-19,12,7) \sigma^{e} & (-1,0,1) \sigma^{e} \\
(-19,12,7) \sigma^{e} & (-54,0,54) \sigma^{e} & (-7,-12,19) \sigma^{e} \\
(-1,0,1) \sigma^{e} & (-7,-12,19) \sigma^{e} & (-2,-8,10) \sigma^{e}
\end{array}\right], \\
C_{i j}^{e}= & \int_{0}^{h} Q_{i}^{\prime} Q_{j}^{\prime \prime} d \xi=\frac{2}{h^{2}}\left[\begin{array}{cccc}
-1 & 2 & -1 \\
0 & 0 & 0 \\
1 & -2 & 1
\end{array}\right] .
\end{aligned}
$$

Assembling all contributions coming from all the elements, Eq. (2.5) yields the system of equations

$$
\begin{aligned}
& A \dot{\delta}-6 a B(\delta) \delta-2 b \widetilde{B}(\sigma) \sigma+a C \delta=0 \\
& A \dot{\sigma}+3 B(\delta) \sigma-C \sigma=0
\end{aligned}
$$

where $\delta=\left(\delta_{-1}, \delta_{0}, \ldots, \delta_{N-1}, \delta_{N}\right)^{T}$ and $\sigma=\left(\sigma_{-1}, \sigma_{0}, \ldots, \sigma_{N-1}, \sigma_{N}\right)^{T}$, and $A$, $B, \widetilde{B}$ and $C$ are $(N+2) \times(N+2)$ global matrices with a generalized $m^{\text {th }}$ row as follows, respectively

$$
\begin{array}{ll}
A: & \frac{h}{30}(1,26,66,26,1), \quad C: \quad \frac{2}{h^{2}}(1,-2,0,2,-1), \\
B: & \frac{1}{30}\left(\begin{array}{l}
(-1,-7,-2,0,0) \delta,(0,-31,-62,-7,0) \delta,(1,31,0,-31,-1) \delta, \\
(0,7,62,31,0) \delta,(0,0,2,7,1) \delta
\end{array}\right), \\
\widetilde{B}: & \frac{1}{30}\left(\begin{array}{l}
(-1,0,1,0,0) \sigma,(-7,-31,31,7,0) \sigma,(-2,-62,0,62,2) \sigma, \\
(0,-7,-31,31,7) \sigma,(0,0,-1,0,1) \sigma
\end{array}\right),
\end{array}
$$


where $\delta=\left(\delta_{m-2}, \delta_{m-1}, \delta_{m}, \delta_{m+1}, \delta_{m+2}\right)^{T}$ and $\sigma=\left(\sigma_{m-2}, \sigma_{m-1}, \sigma_{m}, \sigma_{m+1}\right.$, $\left.\sigma_{m+2}\right)^{T}$.

By substituting the Crank-Nicolson approaches

$$
\delta=0.5\left(\delta^{n}+\delta^{n+1}\right), \quad \sigma=0.5\left(\sigma^{n}+\sigma^{n+1}\right)
$$

and the forward finite difference approximations

$$
\dot{\delta}=\left(\delta^{n+1}-\delta^{n}\right) / \Delta t, \quad \dot{\sigma}=\left(\sigma^{n+1}-\sigma^{n}\right) / \Delta t
$$

into Eq. (2.7), we obtain

$$
\begin{aligned}
& {\left[A-\frac{a \Delta t}{2}(6 B(\delta)-C)\right] \delta^{n+1}-[b \Delta t \widetilde{B}(\sigma)] \sigma^{n+1}} \\
& =\left[A+\frac{a \Delta t}{2}(6 B(\delta)-C)\right] \delta^{n}+[b \Delta t \widetilde{B}(\sigma)] \sigma^{n}, \\
& {\left[A+\frac{\Delta t}{2}(3 B(\delta)-C)\right] \sigma^{n+1}=\left[A-\frac{\Delta t}{2}(3 B(\delta)-C)\right] \sigma^{n} .}
\end{aligned}
$$

Using the boundary conditions in (2.8) yields a $2 N \times 2 N$ system of linear equations. The obtained system is easily solved by a variation of Gauss-elimination algorithm. The following inner iterations are applied two or three times at each time to improve the accuracy of the approximation

$$
\left(\delta^{*}\right)^{n+1}=\delta^{n}+\frac{1}{2}\left(\delta^{n+1}-\delta^{n}\right), \quad\left(\sigma^{*}\right)^{n+1}=\sigma^{n}+\frac{1}{2}\left(\sigma^{n+1}-\sigma^{n}\right) .
$$

Using the relations

$$
U_{N}(x, 0)=\sum_{j=-1}^{N} \delta_{j}^{0} Q_{j}, \quad V_{N}(x, 0)=\sum_{j=-1}^{N} \sigma_{j}^{0} Q_{j}
$$

together with extra conditions, which can be obtained from $U^{\prime}\left(x_{0}, 0\right)=$ $U_{N}^{\prime}\left(x_{0}, 0\right)$ and $V^{\prime}\left(x_{0}, 0\right)=V_{N}^{\prime}\left(x_{0}, 0\right)$, since the first derivative of the approximate initial conditions shall agree with those of the exact initial conditions, initial vector $\delta_{j}^{0}$ can be obtained from the following system of linear equations:

$$
\left[\begin{array}{cccccccc}
\frac{-2}{h} & \frac{2}{h} & & & & & & \\
1 & 1 & & & & & \\
& 1 & 1 & & & & \\
& & & \cdot & & & & \\
& & & & \cdot & & & \\
& & & & & \cdot & & \\
& & & & & 1 & 1
\end{array}\right]\left[\begin{array}{c}
\delta_{-1} \\
\delta_{0} \\
\delta_{1} \\
\cdot \\
\cdot \\
\cdot \\
\delta_{N}
\end{array}\right]=\left[\begin{array}{c}
U_{0}^{\prime} \\
U_{0} \\
U_{1} \\
\cdot \\
\cdot \\
\cdot \\
U_{N}
\end{array}\right],
$$

which can be solved using an appropriate algorithm. A similar system of equation with the same matrix is solved for $\sigma_{j}^{0}$.

Hence, the approximate solution functions for $U(x, t)$ and $V(x, t)$ can be obtained from $\delta^{n}$ and $\sigma^{n}$ using Eq. (2.8). 


\subsection{Stability analysis}

For stability analysis, it is convenient to use the Fourier stability analysis. Since system (2.8) consists of two equations with two variables, we use the following Fourier modes [26]:

$$
\delta_{m}^{n}=P q^{n} e^{i m \varphi}, \quad \sigma_{m}^{n}=W q^{n} e^{i m \varphi},
$$

where $i=\sqrt{-1}, q$ is a complex number, $\varphi$ is a real number and $P, W$ are harmonic amplitudes. For the stability, the condition $|q| \leq 1$ must be satisfied.

To linearize the coupled $\mathrm{KdV}$ equation, we assume that the quantity $U$ and $V$ in the nonlinear terms $U U_{x}$ and $V V_{x}$ are local constants, namely $\widehat{U}$ and $\widehat{V}$, respectively. So, the generalized $m^{\text {th }}$ row of the global matrices $B$ and $\widetilde{B}$ are $\frac{\widehat{U}}{3}(-1,-10,0,10,1)$ and $\frac{\widehat{V}}{3}(-1,-10,0,10,1)$, respectively.

The generalized $m^{\text {th }}$ rows of Eq. (2.8) are

$$
\begin{aligned}
& \alpha_{1} \delta_{m-2}^{n+1}+\alpha_{2} \delta_{m-1}^{n+1}+\alpha_{3} \delta_{m}^{n+1}+\alpha_{4} \delta_{m+1}^{n+1}+\alpha_{5} \delta_{m+2}^{n+1}+\gamma_{4} \sigma_{m-2}^{n+1}+10 \gamma_{4} \sigma_{m-1}^{n+1} \\
& \quad-10 \gamma_{4} \sigma_{m+1}^{n+1}-\gamma_{4} \sigma_{m+2}^{n+1}=\alpha_{5} \delta_{m-2}^{n}+\alpha_{4} \delta_{m-1}^{n}+\alpha_{3} \delta_{m}^{n}+\alpha_{2} \delta_{m+1}^{n}+\alpha_{1} \delta_{m+2}^{n} \\
& \quad-\gamma_{4} \sigma_{m-2}^{n}-10 \gamma_{4} \sigma_{m-1}^{n}+10 \gamma_{4} \sigma_{m+1}^{n}+\gamma_{4} \sigma_{m+2}^{n}
\end{aligned}
$$

and

$$
\begin{aligned}
& \beta_{1} \sigma_{m-2}^{n+1}+\beta_{2} \sigma_{m-1}^{n+1}+\beta_{3} \sigma_{m}^{n+1}+\beta_{4} \sigma_{m+1}^{n+1}+\beta_{5} \sigma_{m+2}^{n+1} \\
& \quad=\beta_{5} \sigma_{m-2}^{n}+\beta_{4} \sigma_{m-1}^{n}+\beta_{3} \sigma_{m}^{n}+\beta_{2} \sigma_{m+1}^{n}+\beta_{1} \sigma_{m+2}^{n},
\end{aligned}
$$

where

$$
\begin{aligned}
& \gamma_{1}=\frac{h}{30}, \quad \gamma_{2}=\frac{\widehat{U} \Delta t}{6}, \quad \gamma_{3}=\frac{\Delta t}{h^{2}}, \quad \gamma_{4}=\frac{b \widehat{V} \Delta t}{3}, \\
& \alpha_{1}=\gamma_{1}+6 \gamma_{2} a+\gamma_{3} a, \quad \alpha_{2}=26 \gamma_{1}+60 \gamma_{2} a-2 \gamma_{3} a, \quad \alpha_{3}=66 \gamma_{1}, \\
& \alpha_{4}=26 \gamma_{1}-60 \gamma_{2} a+2 \gamma_{3} a, \quad \alpha_{5}=\gamma_{1}-6 \gamma_{2} a-\gamma_{3} a, \\
& \beta_{1}=\gamma_{1}-3 \gamma_{2}-\gamma_{3}, \quad \beta_{2}=26 \gamma_{1}-30 \gamma_{2}+2 \gamma_{3}, \quad \beta_{3}=66 \gamma_{1}, \\
& \beta_{4}=26 \gamma_{1}+30 \gamma_{2}-2 \gamma_{3}, \quad \beta_{5}=\gamma_{1}+3 \gamma_{2}+\gamma_{3} .
\end{aligned}
$$

Substituting the Fourier modes given by Eq. (2.9) into (2.10) and (2.11) leads to the following homogeneous algebraic equation system:

$$
\begin{aligned}
& \left.\left(\rho_{1}+c_{1}\right) q-\left(\rho_{1}-c_{1}\right)\right] P+\left[\left(\rho_{4} q+\rho_{4}\right)\right] W=0, \\
& \left.\left(\rho_{1}+c_{2}\right) q-\left(\rho_{1}-c_{2}\right)\right] W=0,
\end{aligned}
$$

where

$$
\begin{aligned}
& \rho_{1}=(66+2 \cos (2 \varphi)+52 \cos (\varphi)) \gamma_{1}, \quad \rho_{2}=(-12 \sin (2 \varphi)-120 \sin (\varphi)) a \gamma_{2}, \\
& \rho_{3}=(-2 \sin (2 \varphi)+4 \sin (\varphi)) a \gamma_{3}, \quad \rho_{4}=(-2 \sin (2 \varphi)-20 \sin (\varphi)) \gamma_{4}, \\
& \rho_{5}=(6 \sin (2 \varphi)+60 \sin (\varphi)) \gamma_{2}, \quad \rho_{6}=(2 \sin (2 \varphi)-4 \sin (\varphi)) \gamma_{3}, \\
& c_{1}=\rho_{2}+\rho_{3}, \quad c_{2}=\rho_{5}+\rho_{6} .
\end{aligned}
$$


For the system (2.12) to have a solution other than zero, the determinant of the coefficient matrix of the system must be zero. Therefore, we get

$$
\left(\rho_{1}^{2}+i \rho_{1} c_{1}+i \rho_{1} c_{2}-c_{1} c_{2}\right) q^{2}-\left(2 \rho_{1}^{2}+2 c_{1} c_{2}\right) q+\rho_{1}^{2}-i \rho_{1} c_{1}-i \rho_{1} c_{2}-c_{1} c_{2}=0
$$

where

$$
q_{1}=\frac{\rho_{1}-i c_{1}}{\rho_{1}+i c_{1}}, \quad q_{2}=\frac{\rho_{1}-i c_{2}}{\rho_{1}+i c_{2}} .
$$

Clearly, the conditions $\left|q_{1}\right| \leq 1$ and $\left|q_{2}\right| \leq 1$ are satisfied. Therefore, the linearized approximations given by Eqs. (2.10) and (2.11) are unconditionally stable.

\section{$3 \quad$ Numerical Examples and Results}

The importance to construct discrete schemes for which the discrete versions of conservation laws are satisfied is well known for many mathematical models. We note nonlinear Schrödinger problems, where discrete conservation laws are playing a very important role in the analysis of stability and accuracy of numerical approximations, see [8]. All numerical calculations were executed on a Pentium i7 PC in the Fortran code using double precision arithmetic. We use the $L_{2}$ and $L_{\infty}$ error norms defined by

$$
\begin{gathered}
L_{2}=\left\|U-U_{N}\right\|_{2}=\sqrt{h \sum_{j=0}^{N}\left|U_{j}-\left(U_{N}\right)_{j}\right|^{2}}, \\
L_{\infty}=\left\|U-U_{N}\right\|_{\infty}=\max _{0 \leq j \leq N}\left|U_{j}-\left(U_{N}\right)_{j}\right|
\end{gathered}
$$

and evaluate only two constants of motion to validate the conservation properties [16]

$$
I_{1}=\int_{-\infty}^{\infty} U d x, \quad I_{2}=\int_{-\infty}^{\infty}\left(U^{2}+\frac{2}{3} b V^{2}\right) d x .
$$

To implement the performance of the method, the following three test problems have been considered: The motion of a single soliton, the interaction of two solitons and birth of solitons.

\subsection{The motion of a single soliton}

We first consider Eq. (1.1) with the homogeneous boundary conditions (1.2) and the initial conditions

$$
U(x, 0)=2 \lambda^{2} \operatorname{sech}^{2}(\xi), \quad V(x, 0)=\frac{1}{2 \sqrt{\omega}} \operatorname{sech}(\xi) .
$$

The exact solution of this problem is [13]

$$
U(x, t)=2 \lambda^{2} \operatorname{sech}^{2}(\xi), \quad V(x, t)=\frac{1}{2 \sqrt{\omega}} \operatorname{sech}(\xi),
$$


Table 1. The numerical solutions of single soliton with $a=0.5, b=-3, \lambda=0.5$ and $\Delta t=0.01$.

\begin{tabular}{cccccccc}
\hline & & & & \multicolumn{2}{c}{$U_{N}(x, t)$} & \multicolumn{2}{c}{$V_{N}(x, t)$} \\
\hline$h$ & $t$ & $I_{1}$ & $I_{2}$ & $L_{2} \times 10^{3}$ & $L_{\infty} \times 10^{3}$ & $L_{2} \times 10^{3}$ & $L_{\infty} \times 10^{3}$ \\
\hline 0.2 & 0.0 & 2.000000 & -0.333333 & 0.000000 & 0.000000 & 0.000000 & 0.000000 \\
& 5.0 & 1.999999 & -0.333333 & 0.021111 & 0.013470 & 0.009392 & 0.004447 \\
& 10.0 & 2.000002 & -0.333333 & 0.043768 & 0.025648 & 0.019462 & 0.010740 \\
& 15.0 & 1.999996 & -0.333332 & 0.065471 & 0.037995 & 0.026306 & 0.014083 \\
& 20.0 & 2.000012 & -0.333331 & 0.056729 & 0.029653 & 0.029793 & 0.011300 \\
\hline \multirow{2}{*}{0.1} & 0.0 & 2.000000 & -0.333333 & 0.000000 & 0.000000 & 0.000000 & 0.000000 \\
& 5.0 & 2.000000 & -0.333334 & 0.001653 & 0.000871 & 0.005180 & 0.002787 \\
& 10.0 & 1.999999 & -0.333333 & 0.005296 & 0.003500 & 0.008284 & 0.004428 \\
& 15.0 & 2.000000 & -0.333333 & 0.008642 & 0.006090 & 0.012757 & 0.006106 \\
& 20.0 & 2.000002 & -0.333332 & 0.005222 & 0.003439 & 0.019473 & 0.009960 \\
\hline
\end{tabular}

where

$$
\xi=\lambda\left(x-\lambda^{2} t\right)+\frac{1}{2 \log (\omega)}, \quad \omega=\frac{-b}{8(4 a+1) \lambda^{4}} .
$$

We will solve the above problem in the interval $-25 \leq x \leq 25$ by taking the following values of $a, b$ and $\lambda$ to compare our results with the earlier works.

Case (a): $a=0.5, b=-3$ and $\lambda=0.5$.

Case (b): $a=-0.5, b=3$ and $\lambda=0.5$.

Case (c): $a=-0.125, b=-3$ and $\lambda=0.5$.

For these three cases the computed values of the invariants $I_{1}$ and $I_{2}$ with the error norms $L_{2}$ and $L_{\infty}$ are presented respectively at some selected times for $h=0.2,0.1$ and $\Delta t=0.01$ in Tables $1-3$. All tables confirm that the error norms $L_{2}$ and $L_{\infty}$ are still small when the time is increased up to $t=20$. The values of the invariants $I_{1}$ and $I_{2}$ remain almost constant during the computer run. For example, the relative change of the invariants $I_{1}$ and $I_{2}$ are respectively $0.093 \times 10^{-3} \%$ and $0.415 \times 10^{-3} \%$ for Case (a), $0.070 \times 10^{-3} \%$ and $0.103 \times 10^{-3 \%}$ for Case (b), $0.057 \times 10^{-3} \%$ and $0.140 \times 10^{-3} \%$ for Case (c) with $\Delta t=0.01$ and $h=0.1$.

Table 4 displays the values of $x$ and amplitudes of $U_{N}$ and $V_{N}$ at different values of $t$. It is clearly seen from the table that the computed values of the amplitudes are very close to their exact ones. It is also observed from the table that the soliton moves to the right at an almost unchanged amplitude with an increasing of time, as expected.

Figure 1 shows the profiles of single solitons at $t=0,10$ and 20 with the error distributions of $U_{N}$ and $V_{N}$. It is also clearly seen from the figure that the soliton moves to the right at an almost unchanged amplitude with an increasing of time as mentioned above and the error distributions are high around the right boundary for $V_{N}$ about the position $x$ at which $U_{N}$ attains its highest amplitude.

Table 5 displays a comparison of the values of the invariants and the error norm $L_{\infty}$ obtained by the present method with those obtained by the quintic 
Table 2. The numerical solutions of single soliton with $a=-0.5, b=3, \lambda=0.5$ and $\Delta t=0.01$.

\begin{tabular}{cccccccc}
\hline & & & & \multicolumn{2}{c}{$U_{N}(x, t)$} & \multicolumn{2}{c}{$V_{N}(x, t)$} \\
\hline$h$ & $t$ & $I_{1}$ & $I_{2}$ & $L_{2} \times 10^{3}$ & $L_{\infty} \times 10^{3}$ & $L_{2} \times 10^{3}$ & $L_{\infty} \times 10^{3}$ \\
\hline 0.2 & 0.0 & 2.000000 & 1.000000 & 0.000000 & 0.000000 & 0.000000 & 0.000000 \\
& 5.0 & 2.000001 & 1.000000 & 0.008026 & 0.005741 & 0.004186 & 0.001814 \\
& 10.0 & 1.999999 & 1.000000 & 0.010025 & 0.004786 & 0.007551 & 0.003250 \\
& 15.0 & 2.000001 & 1.000000 & 0.019918 & 0.012000 & 0.011007 & 0.004569 \\
& 20.0 & 1.999997 & 0.999999 & 0.023566 & 0.014667 & 0.017011 & 0.008244 \\
\hline \multirow{2}{*}{0.1} & 0.0 & 2.000000 & 1.000000 & 0.000000 & 0.000000 & 0.000000 & 0.000000 \\
& 5.0 & 2.000000 & 1.000000 & 0.001570 & 0.000777 & 0.003150 & 0.002053 \\
& 10.0 & 1.999999 & 1.000000 & 0.002180 & 0.001304 & 0.005177 & 0.002665 \\
& 15.0 & 1.999999 & 0.999999 & 0.002939 & 0.001294 & 0.008701 & 0.004710 \\
& 20.0 & 1.999999 & 0.999999 & 0.003737 & 0.001719 & 0.015142 & 0.008143 \\
\hline
\end{tabular}

Table 3. The numerical solutions of single soliton with $a=-0.125, b=-3, \lambda=0.5$ and $\Delta t=0.01$.

\begin{tabular}{cccccccc}
\hline & & & \multicolumn{2}{c}{$U_{N}(x, t)$} & \multicolumn{2}{c}{$V_{N}(x, t)$} \\
\hline$h$ & $t$ & $I_{1}$ & $I_{2}$ & $L_{2} \times 10^{3}$ & $L_{\infty} \times 10^{3}$ & $L_{2} \times 10^{3}$ & $L_{\infty} \times 10^{3}$ \\
\hline 0.2 & 0.0 & 2.000000 & 0.500000 & 0.000000 & 0.000000 & 0.000000 & 0.000000 \\
& 5.0 & 2.000000 & 0.500000 & 0.002851 & 0.001822 & 0.002566 & 0.001309 \\
& 10.0 & 2.000000 & 0.500000 & 0.004240 & 0.002488 & 0.004368 & 0.002211 \\
& 15.0 & 2.000001 & 0.500001 & 0.007778 & 0.004328 & 0.007140 & 0.003498 \\
& 20.0 & 2.000001 & 0.500001 & 0.012679 & 0.006891 & 0.012727 & 0.006340 \\
\hline \multirow{2}{*}{0.1} & 0.0 & 2.000000 & 0.500000 & 0.000000 & 0.000000 & 0.000000 & 0.000000 \\
& 5.0 & 1.999999 & 0.500000 & 0.000991 & 0.000431 & 0.002202 & 0.001269 \\
& 10.0 & 1.999999 & 0.500000 & 0.001486 & 0.000705 & 0.004038 & 0.002272 \\
& 15.0 & 1.999999 & 0.499999 & 0.001870 & 0.000864 & 0.006696 & 0.003590 \\
& 20.0 & 1.999999 & 0.499999 & 0.002646 & 0.001337 & 0.011751 & 0.006171 \\
\hline
\end{tabular}

Table 4. Computed values of position and amplitude for single soliton with $a=0.5$, $b=-3, h=0.1$ and $\Delta t=0.01$.

\begin{tabular}{cccccc}
\hline & & \multicolumn{2}{c}{ Amplitude } & \multicolumn{2}{c}{ Amplitude } \\
\hline$t$ & Position $(x)$ & $U_{N}$ & $U$ & $V_{N}$ & $V$ \\
\hline 0.0 & -1.4 & 0.499772 & 0.499772 & 0.353473 & 0.353473 \\
5.0 & -0.2 & 0.499993 & 0.499993 & 0.353551 & 0.353551 \\
10.0 & 1.1 & 0.499771 & 0.499772 & 0.353471 & 0.353473 \\
15.0 & 2.3 & 0.499990 & 0.499993 & 0.353550 & 0.353551 \\
20.0 & 3.6 & 0.499775 & 0.499772 & 0.353474 & 0.353473 \\
\hline
\end{tabular}




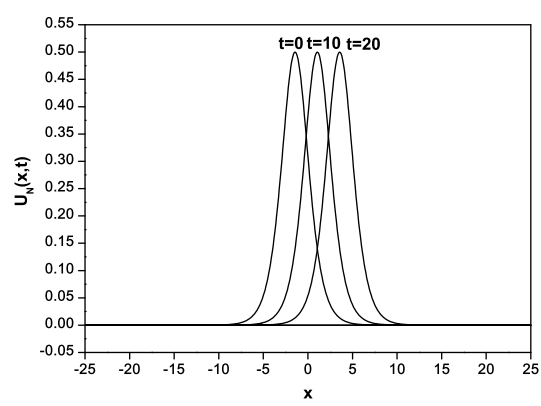

(a)

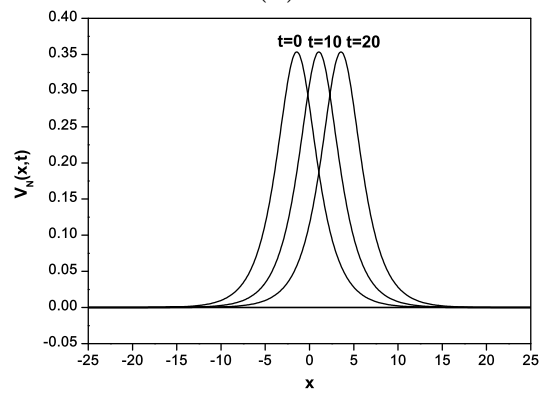

(c)

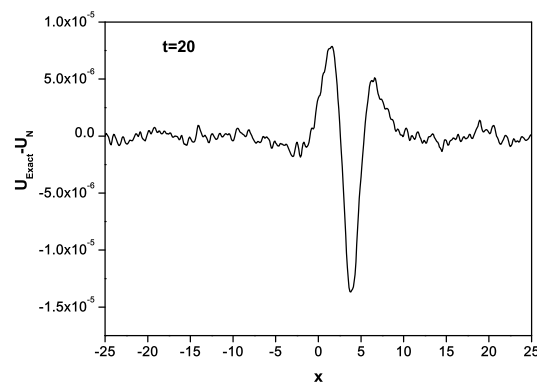

(b)

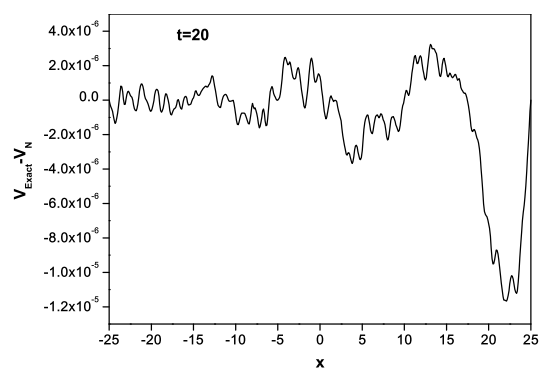

(d)

Figure 1. Single soliton profiles at $t=0,10,20$ and error distributions at $t=20$.

B-spline collocation finite element method given in Ref. [16] at different times for the values of $a, b$ and $\lambda$ given in Cases (a)-(c). It is clearly seen from the table that the invariants are in very good agreement with each other and the present method produces the error norms $L_{\infty}$ at each time smaller than those given in Ref. [16].

\subsection{Interaction of two solitons}

Secondly, the coupled KdV equation (1.1) has been considered with the boundary conditions given by (1.2) and the initial conditions

$$
U(x, 0)=\sum_{j=1}^{2} U_{j}(x, 0), \quad V(x, 0)=\sum_{j=1}^{2} V_{j}(x, 0),
$$

where

$$
\begin{aligned}
& U_{j}(x, 0)=2 \lambda_{j}^{2} \operatorname{sech}^{2}\left(\xi_{j}\right), \quad V_{j}(x, 0)=\frac{1}{2 \sqrt{\omega_{j}} \operatorname{sech}\left(\xi_{j}\right),} \\
& \xi_{j}=\lambda_{j}\left(x-\gamma_{j}\right)+\frac{1}{2 \log \left(\omega_{j}\right)}, \quad \omega_{j}=\frac{-b}{8(4 a+1) \lambda_{j}^{4}} \quad(j=1,2) .
\end{aligned}
$$

For this problem, all calculations are done in the range $-10 \leq x \leq 120$ for $\lambda_{1}=1, \lambda_{2}=0.6, \gamma_{1}=10, \gamma_{2}=30, a=0.5$ and $b=-3$. Tables 6 
Table 5. Comparison of the numerical solutions of the single soliton with results from [16] with $\Delta t=0.01$ and $h=0.1$.

\begin{tabular}{cccccccc}
\hline \multicolumn{7}{c}{ Present Method } & \\
Case & $t$ & $I_{1}$ & $I_{2}$ & $L_{\infty} \times 10^{3}$ & $I_{1}$ & $I_{2}$ & $L_{\infty} \times 10^{3}$ \\
\hline (a) & 0.0 & 2.000000 & -0.333333 & 0.000000 & 2.000000 & -0.333333 & 0.000 \\
& 5.0 & 2.000000 & -0.333334 & 0.000871 & 2.000000 & -0.333333 & 0.004 \\
& 10.0 & 1.999999 & -0.333333 & 0.003500 & 2.000000 & -0.333333 & 0.007 \\
& 15.0 & 2.000000 & -0.333333 & 0.006090 & 2.000000 & -0.333333 & 0.014 \\
& 20.0 & 2.000002 & -0.333332 & 0.003439 & 2.000001 & -0.333333 & 0.026 \\
\hline (b) & 0.0 & 2.000000 & 1.000000 & 0.000000 & 2.000000 & 1.000000 & 0.000 \\
& 5.0 & 2.000000 & 1.000000 & 0.000777 & 2.000000 & 1.000000 & 0.003 \\
& 10.0 & 1.999999 & 1.000000 & 0.001304 & 2.000000 & 1.000000 & 0.003 \\
& 15.0 & 1.999999 & 0.999999 & 0.001294 & 1.999998 & 0.999999 & 0.005 \\
& 20.0 & 1.999999 & 0.999999 & 0.001719 & 1.999999 & 0.999999 & 0.008 \\
\hline (c) & 0.0 & 2.000000 & 0.500000 & 0.000000 & 2.000000 & 0.500000 & 0.000 \\
& 5.0 & 1.999999 & 0.500000 & 0.000431 & 2.000000 & 0.500000 & 0.003 \\
& 10.0 & 1.999999 & 0.500000 & 0.000705 & 1.999999 & 0.500000 & 0.003 \\
& 15.0 & 1.999999 & 0.499999 & 0.000864 & 1.999999 & 0.500000 & 0.003 \\
& 20.0 & 1.999999 & 0.499999 & 0.001337 & 1.999999 & 0.500000 & 0.004 \\
\hline
\end{tabular}

and 7 present a comparison of the calculated conservation constants at different space and time steps. The variations of the invariants $I_{1}$ and $I_{2}$ remain almost constant during the computer run. For example, the relative change of the invariants $I_{1}$ and $I_{2}$ are respectively $0.015 \%$ and $0.264 \%$ with $\Delta t=0.05$ and $h=0.1 ; 4.657 \times 10^{-3 \%}$ and $3.716 \times 10^{-3 \%}$ with $\Delta t=0.02$ and $h=0.1$; $4.278 \times 10^{-3} \%$ and $3.420 \times 10^{-3} \%$ with $\Delta t=0.01$ and $h=0.2 ; 2.005 \times 10^{-3} \%$ and $1.773 \times 10^{-3 \%}$ with $\Delta t=0.01$ and $h=0.1$.

Table 8 displays a comparison of the invariants obtained by the present method with those obtained by the quintic B-spline collocation finite element method given in Ref. [16]. From Table 8, it is clearly seen that both results are in good agreement with each other.

Table 6. Invariants for the interaction of two solitons with $h=0.1$ for $\Delta t=0.02,0.05$.

\begin{tabular}{ccccc}
\hline & \multicolumn{2}{c}{$\Delta t=0.05$} & \multicolumn{2}{c}{$\Delta t=0.02$} \\
\hline$t$ & $I_{1}$ & $I_{2}$ & $I_{1}$ & $I_{2}$ \\
\hline 0.0 & 6.400000 & -3.242667 & 6.400000 & -3.242667 \\
10.0 & 6.399930 & -3.243751 & 6.399999 & -3.242693 \\
20.0 & 6.400085 & -3.244850 & 6.400025 & -3.242688 \\
30.0 & 6.400259 & -3.245970 & 6.400065 & -3.242689 \\
40.0 & 6.398464 & -3.247081 & 6.399711 & -3.242722 \\
50.0 & 6.397809 & -3.248153 & 6.399575 & -3.242711 \\
60.0 & 6.400838 & -3.248617 & 6.400174 & -3.242697 \\
70.0 & 6.401932 & -3.248917 & 6.400376 & -3.242689 \\
80.0 & 6.399879 & -3.249991 & 6.399969 & -3.242711 \\
90.0 & 6.399074 & -3.251013 & 6.399695 & -3.242713 \\
\hline
\end{tabular}


Table 7. Invariants for the interaction of two solitons with $\Delta t=0.01$ for $h=0.1,0.2$.

\begin{tabular}{ccccc}
\hline \multicolumn{2}{c}{$h=0.2$} & \multicolumn{2}{c}{$h=0.1$} \\
\hline$t$ & $I_{1}$ & $I_{2}$ & $I_{1}$ & $I_{2}$ \\
\hline 0.0 & 6.400000 & -3.242667 & 6.400000 & -3.242667 \\
10.0 & 6.399999 & -3.242674 & 6.399998 & -3.242675 \\
20.0 & 6.400071 & -3.242679 & 6.400010 & -3.242662 \\
30.0 & 6.400202 & -3.242677 & 6.400025 & -3.242657 \\
40.0 & 6.398900 & -3.242693 & 6.399881 & -3.242678 \\
50.0 & 6.398441 & -3.242725 & 6.399826 & -3.242689 \\
60.0 & 6.400494 & -3.242631 & 6.400076 & -3.242695 \\
70.0 & 6.401631 & -3.242704 & 6.400174 & -3.242689 \\
80.0 & 6.400228 & -3.242689 & 6.400000 & -3.242678 \\
90.0 & 6.399726 & -3.242778 & 6.399877 & -3.242683 \\
\hline
\end{tabular}

Table 8. Comparison of numerical solutions of the interaction of two solitons with results from [16] with $h=0.1$ and $\Delta t=0.01$.

\begin{tabular}{ccccc}
\hline \multicolumn{2}{c}{ Present Method } & \multicolumn{2}{c}{$[16]$} \\
\hline$t$ & $I_{1}$ & $I_{2}$ & $I_{1}$ & $I_{2}$ \\
\hline 0.0 & 6.400000 & -3.242667 & 6.400000 & -3.243013 \\
10.0 & 6.399998 & -3.242675 & 6.400001 & -3.243012 \\
20.0 & 6.400010 & -3.242662 & 6.399995 & -3.243009 \\
30.0 & 6.400025 & -3.242657 & 6.399946 & -3.243015 \\
40.0 & 6.399881 & -3.242678 & 6.399991 & -3.243102 \\
50.0 & 6.399826 & -3.242689 & 6.399962 & -3.243008 \\
60.0 & 6.400076 & -3.242695 & 6.399863 & -3.243008 \\
70.0 & 6.400174 & -3.242689 & - & - \\
80.0 & 6.400000 & -3.242678 & - & - \\
90.0 & 6.399877 & -3.242683 & - & - \\
\hline
\end{tabular}

The experiment was run from $t=0$ to $t=90$ to allow the interaction of two solitons to take place. Figure 2 shows the interaction of two solitons for $U_{N}$. As it is seen from Figure 2, the amplitudes of the big and small waves at $t=0$ are 1.996731 at the point $x=10.2$ and 0.719566 at the point $x=53.1$, respectively. As time increases, both of the waves move forward the right and then the big wave catches up the smaller one. At $t=90$, it is observed that the big wave leaves from the small one and they both move forward. At $t=90$, the amplitude of the big wave is 1.987528 at the point $x=103.1$ whereas the amplitude of the smaller one is 0.719579 at the point $x=80.9$.

Figure 3 illustrates the behaviour of the interaction of two solitons for $V_{N}$ at times from $t=0$ to $t=90$. At $t=0$, the amplitudes of the big and small waves are 1.413057 at the point $x=10.2$ and 0.508964 at the point $x=53.1$, respectively. As time increases, both of the waves move forward the right and the big wave catches up the smaller one. It is observed that at $t=90$ the big wave leaves from the small one and then they both move forward. At $t=90$, the amplitude of small wave is 0.508966 with having the position $x=80.9$ while the amplitude of the big one is 1.409834 with having the position $x=103.1$. 


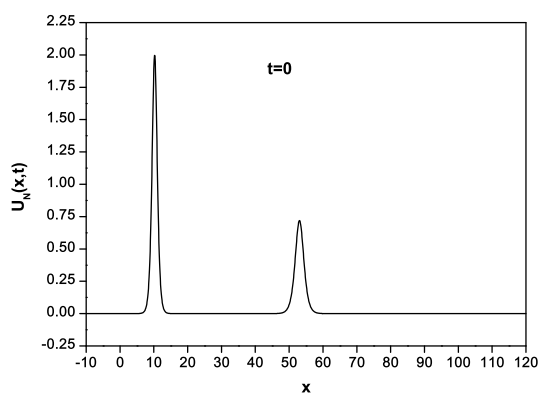

(a)

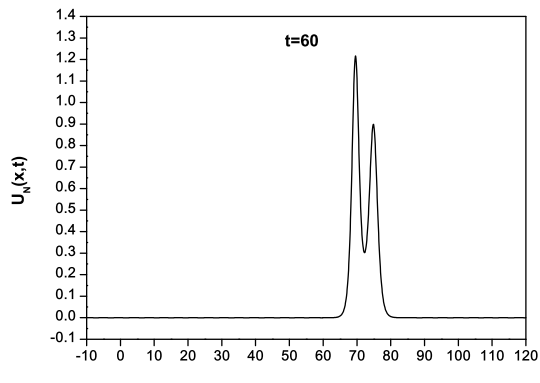

(c)

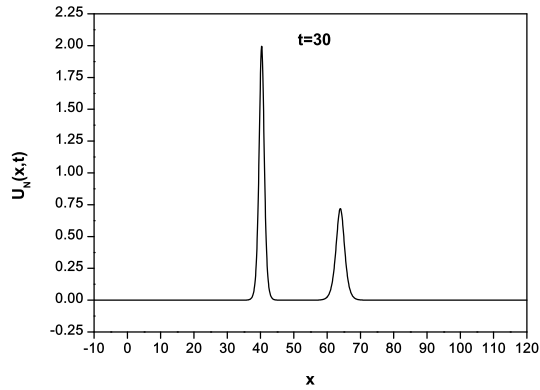

(b)

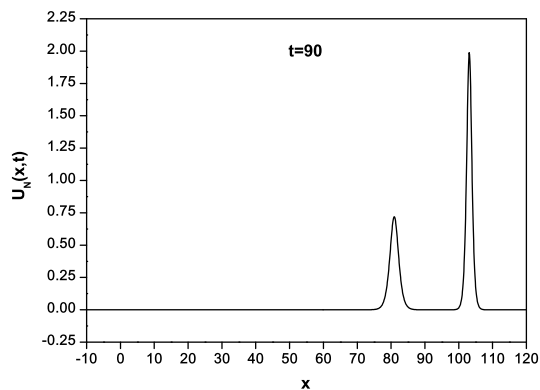

(d)

Figure 2. The interaction of two solitons for $U$ at different times.

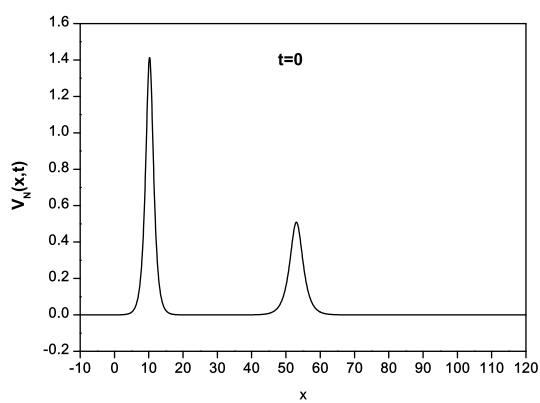

(a)

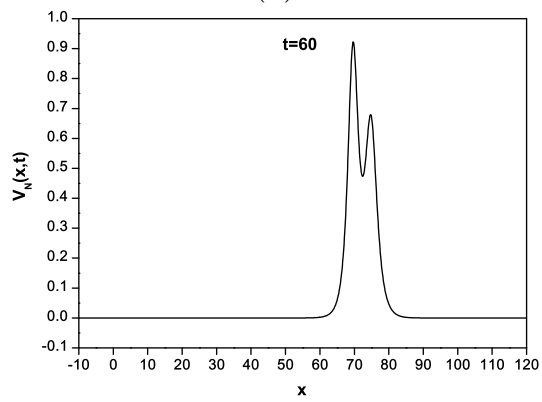

(c)

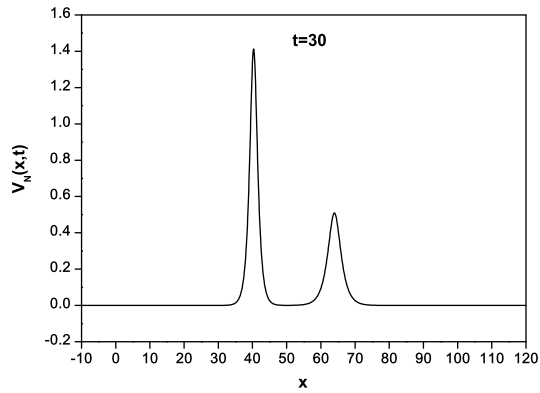

(b)

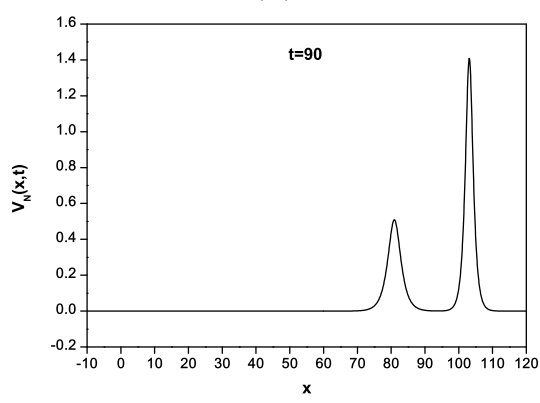

(d)

Figure 3. The interaction of two solitons for $V$ at different times. 
Table 9. The numerical solutions of the birth of soliton and a comparison with those given in Ref. [16].

\begin{tabular}{|c|c|c|c|c|c|c|c|}
\hline \multirow[b]{3}{*}{$\Delta t$} & \multirow[b]{3}{*}{$t$} & \multicolumn{4}{|c|}{ Present Method } & \multirow{2}{*}{\multicolumn{2}{|c|}{$\frac{[16]}{h=0.1}$}} \\
\hline & & \multicolumn{2}{|c|}{$h=0.2$} & \multicolumn{2}{|c|}{$h=0.1$} & & \\
\hline & & $I_{1}$ & $I_{2}$ & $I_{1}$ & $I_{2}$ & $I_{1}$ & $I_{2}$ \\
\hline \multirow[t]{6}{*}{0.02} & 0.0 & 17.724539 & -12.533142 & 17.724539 & -12.533142 & & \\
\hline & 10.0 & 17.724552 & -12.534584 & 17.724517 & -12.533817 & & \\
\hline & 20.0 & 17.724438 & -12.538644 & 17.724436 & -12.537647 & & \\
\hline & 30.0 & 17.723768 & -12.542624 & 17.724235 & -12.541628 & & \\
\hline & 40.0 & 17.727828 & -12.546647 & 17.727083 & -12.545628 & & \\
\hline & 50.0 & 17.731455 & -12.550704 & 17.727015 & -12.549471 & & \\
\hline \multirow[t]{6}{*}{0.01} & 0.0 & 17.724539 & -12.533142 & 17.724539 & -12.533142 & 17.72454 & -12.53314 \\
\hline & 10.0 & 17.724575 & -12.533963 & 17.724539 & -12.533214 & 17.72454 & -12.53316 \\
\hline & 20.0 & 17.724568 & -12.534382 & 17.724528 & -12.533502 & 17.72454 & -12.53316 \\
\hline & 30.0 & 17.724072 & -12.534597 & 17.724476 & -12.533695 & 17.72449 & -12.53320 \\
\hline & 40.0 & 17.727303 & -12.534727 & 17.725440 & -12.533943 & 17.72448 & -12.53321 \\
\hline & 50.0 & 17.729995 & -12.535070 & 17.725238 & -12.533980 & 17.72469 & -12.53320 \\
\hline
\end{tabular}

As a result, Figures 2 and 3 show the interaction of two positive amplitude waves having the same position at the point $x$ and at the same time $t$ having different amplitudes.

\subsection{Birth of solitons}

As a final problem, we consider the coupled $\mathrm{KdV}$ equation given by (1.1) with the initial conditions

$$
U(x, 0)=e^{-0.01 x^{2}}, \quad V(x, 0)=e^{-0.01 x^{2}}
$$

and the boundary conditions (1.2).

All calculations for this problem are done in the range $-50 \leq x \leq 150$ for the values $a=0.5$ and $b=-3$. The experiment was run from $t=0$ to $t=50$. The values of the invariants obtained by the present method at different space and time steps are displayed in Table 9. The table also presents a comparison of the invariants obtained by the present method with those given in Ref. [16]. It is obviously seen from the table that the results are in good agreement with each other.

It is clearly seen from the table that the variations of the invariants $I_{1}$ and $I_{2}$ are satisfactorily well. For example, the relative change of the invariants $I_{1}$ and $I_{2}$ are respectively $0.039 \%$ and $0.140 \%$ with $\Delta t=0.02$ and $h=0.2 ; 0.014 \%$ and $0.130 \%$ with $\Delta t=0.02$ and $h=0.1 ; 0.031 \%$ and $0.015 \%$ with $\Delta t=0.01$ and $h=0.2 ; 3.946 \times 10^{-3 \%}$ and $6.687 \times 10^{-3} \%$ with $\Delta t=0.01$ and $h=0.1$.

The solutions $U_{N}$ and $V_{N}$ obtained for $a=0.5, b=-3$ and $t=0,30$ and 50 are illustrated in Figure 4 and 5, respectively. It is seen from the figures that at $t=0$, there is only a single wave for each of $U_{N}$ and $V_{N}$ with the amplitude 1.0 at $x=0$, and then each single wave for $U_{N}$ and $V_{N}$ moves to the right when time increases and then each one creates a large number of waves behind 


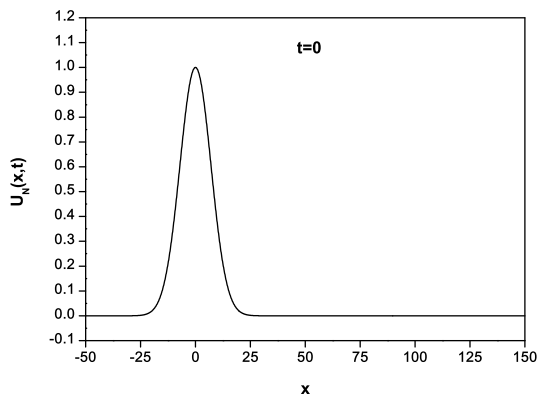

(a)

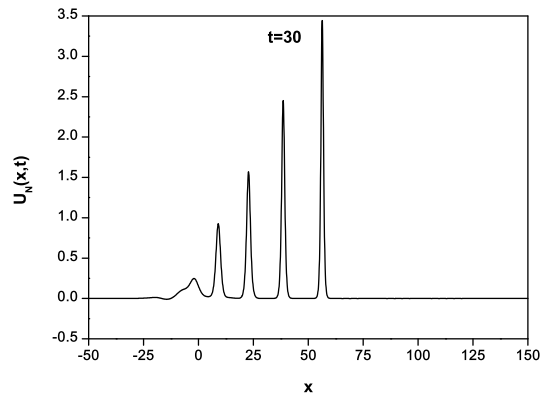

(c)

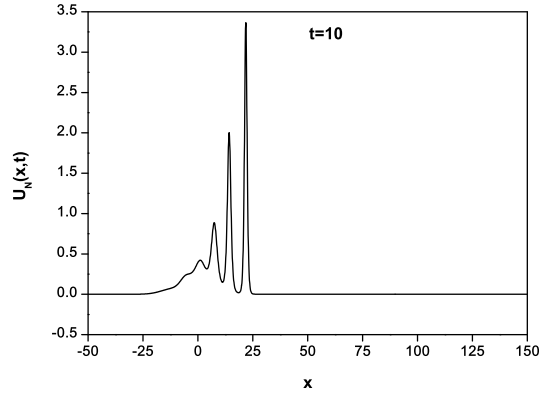

(b)

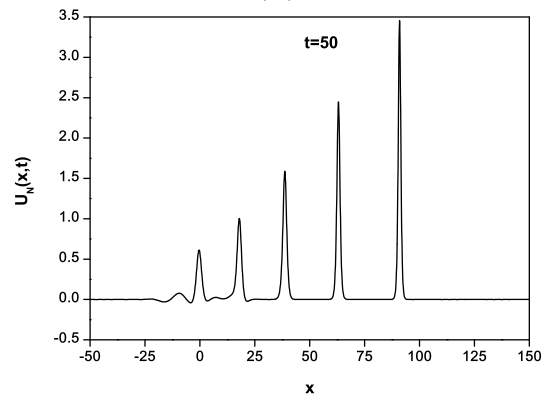

(d)

Figure 4. Solution profiles of the birth of soliton for $U$ at various times.

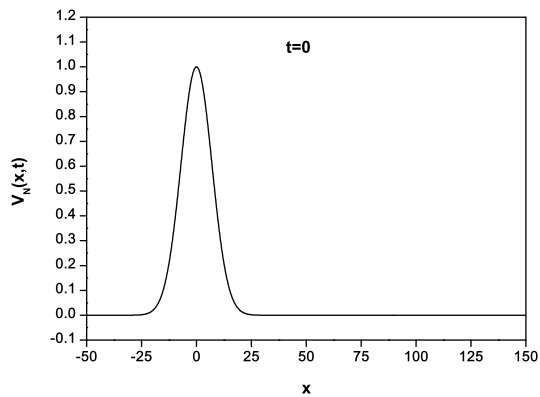

(a)

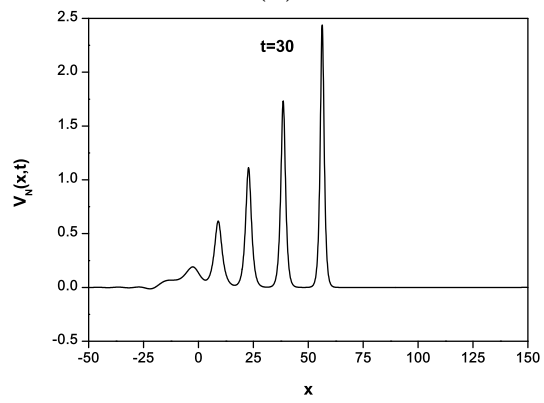

(c)

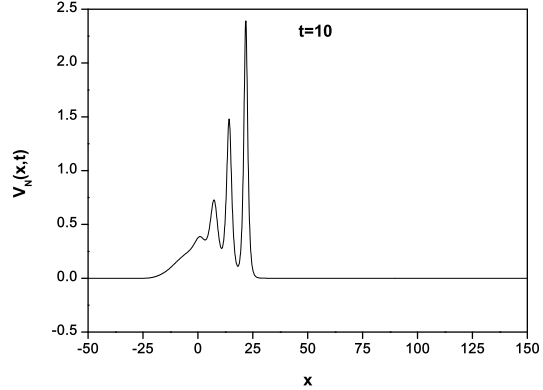

(b)

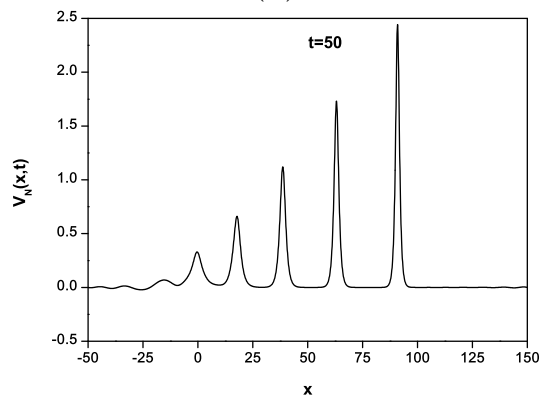

(d)

Figure 5. Solution profiles of the birth of soliton for $V$ at various times. 
Table 10. Computed values of position and amplitude for the birth of soliton with $h=0.1$ and $\Delta t=0.01$ at $t=50$.

\begin{tabular}{ccccc}
\hline & Position $(x)$ & Amplitude $\left(U_{N}\right)$ & $\operatorname{Position}(x)$ & $\operatorname{Amplitude}\left(V_{N}\right)$ \\
\hline First wave & 90.9 & 3.457008 & 90.9 & 2.444029 \\
Second wave & 63.1 & 2.447592 & 63.1 & 1.731882 \\
Third wave & 38.7 & 1.587947 & 38.6 & 1.120752 \\
Fourth wave & 18.0 & 1.004435 & 17.8 & 0.660323 \\
Fifth wave & -0.4 & 0.612468 & -0.4 & 0.329449 \\
\hline
\end{tabular}

itself with different amplitudes at $t=50$. The positions and amplitudes of the successive waves at time $t=50$ are given in Table 10 .

\section{Conclusions}

In this paper, the numerical solutions of the coupled $\mathrm{KdV}$ equation with various initial and boundary conditions were obtained using the Galerkin quadratic Bspline finite element method. The efficiency of the method was tested on three numerical experiments of wave propagation: The motion of a single soliton, the interaction of two solitons and the birth of solitons. The accuracy of the method was examined by the error norms $L_{2}$ and $L_{\infty}$. The obtained results show that the error norms are reasonably small and the conservation properties are all very good. As a conclusion, the method can also be used efficiently for solving a large number of coupled nonlinear partial differential equations.

\section{References}

[1] S. Abbasbandy. The application of homotopy analysis method to solve a generalized Hirota-Satsuma coupled KdV equation. Phys. Lett. A, 361:478-483, 2007. http://dx.doi.org/10.1016/j.physleta.2006.09.105.

[2] A.S. Abdel Rady, E.S. Osman and M. Khalfallah. Multi soliton solution for the system of coupled Korteweg-de Vries equations. Appl. Math. Comput., 210:177181, 2009. http://dx.doi.org/10.1016/j.amc.2008.12.076.

[3] K. Al-Khaled, M. Al-Refai and A. Alawneh. Traveling wave solutions using the variational method and the tanh method for nonlinear coupled equations. Appl. Math. Comput., 202:233-242, 2008.

http://dx.doi.org/10.1016/j.amc.2008.02.009.

[4] B. Alvarez-Samaniego and X. Carvajal. On the local well-posedness for some systems of coupled KdV equations. Nonlinear Anal., 69:692-715, 2008. http://dx.doi.org/10.1016/j.na.2007.06.009.

[5] L.M.B. Assas. Variational iteration method for solving coupled-KdV equations. Chaos Solitons Fractals, 38:1225-1228, 2008. http://dx.doi.org/10.1016/j.chaos.2007.02.012.

[6] A. Biswas and M.S. Ismail. 1-Soliton solution of the coupled KdV equation and Gear-Grimshaw model. Appl. Math. Comput., 216(12):3662-3670, 2010. http://dx.doi.org/10.1016/j.amc.2010.05.017. 
[7] D. Bo Cao, J. Ren Yan and Y. Zhang. Exact solutions for a new coupled MKdV equations and a coupled KdV equations. Phys. Lett. A, 297:68-74, 2002. http://dx.doi.org/10.1016/S0375-9601(02)00376-6.

[8] R. Čiegis, I. Laukaitytė and M. Radziunas. Numerical algorithms for Schrödinger equations with artificial boundary conditions. Nonlinear Functional Analysis and Optimization, 30(9-10):903-923, 2009. http://dx.doi.org/dx.doi.org/10.1080/01630560903393097.

[9] E. Fan. Traveling wave solutions for nonlinear equations using symbolic computation. Comput. Math. Appl., 43:671-680, 2002. http://dx.doi.org/10.1016/S0898-1221(01)00312-1.

[10] E. Fan and H. Zhang. New exact solutions to a system of coupled KdV equations. Phys. Lett. A, 245:389-392, 1998. http://dx.doi.org/10.1016/S0375-9601(98)00464-2.

[11] A.A. Halim, S.P. Kshevetskii and S.B. Leble. On numerical integration of coupled Korteweg-de Vries system. Comput. Math. Appl., 45(4-5):581-591, 2003. http://dx.doi.org/10.1016/S0898-1221(03)00018-X.

[12] A.A. Halim and S.B. Leble. Analytical and numerical solution of coupled KdVMKdV system. Chaos Solitons Fractals, 19:99-108, 2004. http://dx.doi.org/10.1016/S0960-0779(03)00085-7.

[13] R. Hirota and J. Satsuma. Soliton solution of the coupled KdV system. Phys. Lett. A, 85:407-408, 1981. http://dx.doi.org/10.1016/0375-9601(81)90423-0.

[14] I.E. Inan. Exact solutions for coupled KdV equation and $\mathrm{KdV}$ equations. Phys. Lett. A, 371:90-95, 2007. http://dx.doi.org/10.1016/j.physleta.2007.06.009.

[15] S. Islam, S. Haq and M. Uddin. A mesh free interpolation method for the numerical solution of the coupled nonlinear partial differential equations. Eng. Anal. Bound. Elem., 33:399-409, 2009.

http://dx.doi.org/10.1016/j.enganabound.2008.06.005.

[16] M.S. Ismail. Numerical solution of a coupled Korteweg-de Vries equations by collocation method. Numer. Methods Partial Differential Equations, 25(2):275291, 2009. http://dx.doi.org/10.1002/num.20343.

[17] M. Ito. Symmetries and conservation laws of a coupled nonlinear wave equations. Phys. Lett. A, 91:335-338, 1982.

http://dx.doi.org/10.1016/0375-9601(82)90426-1.

[18] A. Karasu (Kalkanli) and T. Kilic. Integrability of a nonautonomous coupled KdV system. IJMPC, 15(5):609-617, 2004.

[19] D. Kaya and I.E. Inan. Exact and numerical traveling wave solutions for nonlinear coupled equations using symbolic computation. Appl. Math. Comput., 151:775-787, 2004. http://dx.doi.org/10.1016/S0096-3003(03)00535-6.

[20] P. Kumar Roy. On coupled KdV equations. Phys. Lett. A, 249:55-58, 1998. http://dx.doi.org/10.1016/S0375-9601(98)00716-6.

[21] Z. Ma and J. Zhu. Jacobian elliptic function expansion solutions for the Wicktype stochastic coupled KdV equations. Chaos Solitons Fractals, 32:1679-1685, 2007. http://dx.doi.org/10.1016/j.chaos.2005.11.085.

[22] R. Mokhtari and M. Mohammadi. New exact solutions to a class of coupled nonlinear PDEs. Int. J. Nonlinear Sci. Numer. Simul., 10(6):779-796, 2009. http://dx.doi.org/10.1515/IJNSNS.2009.10.6.779. 
[23] P.M. Prenter. Splines and Variational Methods. Wiles, New York, 1975.

[24] S. Qian and L. Tian. Nonlocal Lie-Backlund symmetries of the coupled KdV system. Phys. Lett. A, 364:235-238, 2007. http://dx.doi.org/10.1016/j.physleta.2006.12.008.

[25] A. Rashid and A.I.B.M. Ismail. Error estimates of spectral collocation method for the coupled Korteweg-de Vries equations. J. Appl. Funct. Anal., 6(1):17-25, 2011.

[26] M. Shashkov. Conservative Finite Difference Methods on General Grids. CRC, USA, 1996.

[27] H.W. Tam, W. Ma, X.-B. Hu and D. Wang. The Hirota-Satsuma coupled KdV equation and coupled Ito system revisited. J. Phys. Soc. Japan, 69:45-52, 2000. http://dx.doi.org/10.1143/JPSJ.69.45.

[28] B. Tian and Y.-T. Gao. Exact solutions for the Bogoyavlenskii coupled KdV equations. Phys. Lett. A, 208:193-196, 1995. http://dx.doi.org/10.1016/0375-9601(95)00737-N.

[29] Y. Zhou, M. Wang and Y. Wang. Periodic wave solutions to a coupled KdV equations with variable coefficients. Phys. Lett. A, 308:31-36, 2003. http://dx.doi.org/10.1016/S0375-9601(02)01775-9.

[30] S. Zhu. A difference scheme for the coupled KdV equation. Commun. Nonlinear Sci. Numer. Simul., 4(1):60-63, 1999. http://dx.doi.org/10.1016/S1007-5704(99)90059-X. 